\title{
USE OF BIOLOGICAL THERAPY IN CHRONIC ARTICULAR CONDITION AFTER CHIKUNGUNYA INFECTION: CASE REPORT
}

\author{
Bruna Luiza Oliveira Lima1, ${ }^{1}$, Cristyan César Dall Agnol ${ }^{1}$, Mirian Parolo Ribeiro ${ }^{1}$, Raiane Alves de Matos ${ }^{1}$, Camila de Oliveira
} Trevisan Coutinho ${ }^{1}$

1. Universidade Federal de Mato Grosso, Sinop (MT), Brazil.

*Corresponding author: bbrunalima99@gmail.com

\section{BACKGROUND}

Chikungunya is a viral illness caused by chikungunya virus (CHIKV) and has become a relevant public health problem, as half of the cases tend to evolve into chronic, persistent and disabling arthritis. The signs and symptoms are clinically similar to those of dengue, such as sudden onset high fever, headache and skin rashes. The main clinical manifestation that differentiates them is the severe bilateral joint pain that occurs in chikungunya, with or without edema and may persist for months to years. It should be noted that the data in the literature regarding therapeutic treatment in the various stages of arthropathy caused by CHIKV are limited, but biological therapy has shown promise in cases in which joint inflammatory activity is maintained even after the use of corticosteroids, hydroxychloroquine and methotrexate.

\section{CASE REPORT}

Male patient, 63 years old, reported inflammatory arthralgia and morning stiffness in wrists, ankles and fourth and fifth interphalangeal joints bilaterally, thirty days after presenting fever with myalgia and diffuse rash, with positive serology for chikungunya. After the diagnosis of arthritis secondary to chikungunya, therapy with hydroxychloroquine $400 \mathrm{mg} / \mathrm{day}$ and corticosteroid therapy with prednisone were established. After 2 months, the patient returned with worsening of the arthralgic condition after initial improvement and opted for replacing hydroxychloroquine by methotrexate $15 \mathrm{mg} /$ week. Reassessed after 60 days, the patient presented a slight improvement of the condition and remained with high inflammatory tests, thus opting to increase the dose of methotrexate to 20 $\mathrm{mg} /$ week and maintenance of prednisone. After reassessment and finding that the previous pain persisted, biological therapy with adalimumab 40 mg 14/14 days was associated with treatment, resulting in complete improvement of the joint condition and decrease in inflammatory tests after 2 months of treatment.

\section{CONCLUSION}

Biological therapy, according to recommendations of the Brazilian Society of Rheumatology, can be prescribed in patients with chronic inflammatory joint post-CHIKV infection, refractory to the use of corticosteroids, hydroxychloroquine and methotrexate. However, its use is not recommended in the acute phase, even in patients who already use it to treat another rheumatic disease, and the suspension of treatment with immunobiologicals in these cases is indicated. However, the level of evidence for this therapy is considered low, mainly due to the lack of clinical trials that demonstrate its effectiveness. Therefore, it was possible to observe that the patient with arthritis secondary to chikungunya only obtained significant improvement after the introduction of biological therapy with adalimumab. 